A Standard Analysis Method (SAM) for the Automated Analysis of Polychlorinated Biphenyls (PCBs) in Soils using the Chemical Analysis Automation (CAA) Paradigm:

Validation and Performance

Charles Rzeszutko

C. Robert Johnson

Matthew Monagle

Leon N. Klatt* 


\section{Dischamiex}

Portions of this docoment myy be Illegible in electronic image products. Images are produced from the best available original doermentert 


\section{DISCLAIMER}

This report was prepared as an account of work sponsored by an agency of the United States Government. Neither the United States Government nor any agency thereof, nor any of their employees, make any warranty, express or implied, or assumes any legal liabiity or responsibiity for the accuracy, completeness, or usefulness of any information, apparatus, product, or process disclosed, or represents that its use would not infringe privately owned rights. Reference herein to any specific commercial product, process, or service by trade name, trademark, manufacturer, or otherwise does not necessarily constitute or imply its endorsement, recommendation, or favoring by the United States Government or any agency thereof. The views and opinions of authors expressed herein do not necessar. ily state or reflect those of the United States Government or any agency thereof. 


\title{
A STANDARD ANALYSIS METHOD (SAM) FOR THE AUTOMATED ANALYSIS OF POLYCHLORINATED BIPHENYLS (PCBS) IN SOILS USING THE CHEMICAL ANALYSIS AUTOMATION (CAA) PARADIGM: VALIDATION AND PERFORMANCE
}

\author{
Charles Rzeszutko, C. Robert Johnson, Matthew Monagle, and Leon N. Klatt
}

\begin{abstract}
The Chemical Analysis Automation (CAA) program is developing a standardized modular automation strategy for chemical analysis. In this automation concept, analytical chemistry is performed with modular building blocks that correspond to individual elements of the steps in the analytical process. With a standardized set of behaviors and interactions, these blocks can be assembled in a plug-and-play manner into a complete analysis system. These building blocks, which are referred to as Standard Laboratory Modules (SLM), interface to a host control system that orchestrates the entire analytical process, from sample preparation through data interpretation. The integrated system is called a Standard Analysis Method (SAM).

A SAM for the automated determination of polychlorinated biphenyls (PCBs) in soils, assembled in a mobile laboratory, is undergoing extensive testing and validation. The SAM consists of the following SLMs: a four-channel Soxhlet extractor, a high-volume concentrator, a column clean-up, a gas chromatograph, a PCB data-interpretation module, a robot, and a human-computer interface. The SAM is configured to meet the requirements specified in the U.S. Environmental Protection Agency's (EPA) SW-846 methods $3541 / 3620 A / 8082$ for the analysis of PCBs in soils. The PCB SAM will be described along with the developmental test plan. Performance data obtained during developmental testing will also be discussed.
\end{abstract}




\section{INTRODUCTION}

The U.S. Department of Energy, Office of Science and Technology (DOE/EM-50) began in 1991 to develop a series of standardized, automated laboratory technologies for the analysis of environmental samples. These technologies are referred to collectively as the CAA program. This paper describes the developmental test plan and results to date for the first set of hardware and software technologies produced by the program--those for the analysis of PCBs in soil.

The following CAA program goals apply to a wide range of analytical laboratory procedures:

- reduce analysis cost per sample

- reduce variations in data quality among laboratories

- increase precision and accuracy of results

- reduce frequency and unpredictability of spoiled measurements

- minimize exposure of personnel to hazardous materials

- decrease time between submission of the sample and production of the final laboratory report.

- better utilize skilled analysts, by enabling them to focus on complex rather than routine tasks

Some of these goals could be addressed through improvements to state-of-the-art manual or semiautomated analytical technologies. However, two significant factors led to the CAA program's decision to proceed with a fully automated approach. First, collectively these goals indicate a need for systematic, uninterrupted, and predictable testing beyond what can be envisioned using next-generation manual or even semiautomated laboratory technologies. Second, the CAA program chose to improve sample flow through better integration of the component steps from sample preparation through report generation. Complete "end-to-end integration" that reduces substantially the time between the component steps is better achieved by automation than by manual transport of the samples.

Both the hardware modules ancl software modules are called Standard Laboratory Modules (SLMs). When hardware and software SLMs are assembled together to perform a complete laboratory analysis (for example, a designated EPA method), the ensemble is called a Standard Analysis Method (SAM). The nomenclature reflects the CAA program's design objective of standardized, modular equipment that can be used either stand-alone or readily integrated with one another. The modularity of the SLMs and the use of a common communications protocol means that a set of SLMs can be arranged into a variety of different combinations. A detailed overview and description of the CAA program is described in the paper by Hollen. ${ }^{1}$

To date the CAA program includes 26 hardware modules and eight software modules. Most of the modules were DOE-developed (either at the national laboratories or through support of university research). Others were purchased off-the-shelf and subsequently modified. 
A fully automated PCB SAM was successfully integrated into the CAA program's Mobile Environmental Laboratory (MEL) testbed during the summer of 1996.

Preliminary testing and performance evaluation of the PCB SAM started in September 1996 at Oak Ridge National Laboratory (ORNL), where the MEL is currently sited. The CAA program, in cooperation with the Institute for Defense Analyses, designed a developmental test plan for the PCB SAM. These developmental tests are intended largely to validate that the SLM modules meet the design specifications for chemical performance. At the same time, important information about the flow of work (labor, consumables, schedule, etc.) should be investigated. Developmental test results provide early indicators about the cost and performance of the system - information too important to be overlooked. ${ }^{2}$

\section{EXPERIMENTAL}

\section{System Description}

Hardware SLMs to be tested include a four-channel Soxhlet extractor; a high-volume concentrator (HVC); a clean-up column module consisting of a Gilson syringe pump Model 402 and a sampling injector Model 231XL (Gilson Medical Electronics, Inc., Middleton, WI); and a Varian Model $3400 \mathrm{CX}$ gas chromatograph with electron capture detector equipped with a Model 8000 liquid autosampler (Varian, Walnut Creek, CA). Samples are carried among the SLMs by an ORCA robot (Sagian, Inc., Indianapolis, IN) moving along a three-meter linear track. Software SLMs to be tested include the PCB Data Interpretation Module (DIM), the Task Sequence Controller (TSC), and the Human-Computer Interface ( $\mathrm{HCl})$.

\section{Soxhlet Extractor SLM}

The automated Soxhlet SLM performs exactly the same solvent extraction process with four extractors operating in parallel. The analyst weighs and dries a 10-gram soil sample and places it in a cellulose thimble with a metal ring attached to the opening at the top. A robot places the thimble into one of the SLM's four entry ports. The SLM uses a simple video system to confirm that a thimble is indeed present. A magnetic ring is lowered, which picks up the thimble ring. The SLM then retracts the thimble into the column. A clean beaker is placed upon the heating pad, again by a robot, and once the video system has confirmed that the beaker is present, the heating pad and beaker rise automatically until the beaker contacts the bottom of the condensing column. At this point, the SLM needs no further analyst or robot intervention until the extraction is complete. The SLM adds a preset volume of solvent through a supply valve at the top of the condensing column; the solvent flows down the condensing column through the thimble and into the beaker. The SLM lowers the thimble into the solvent and power is supplied to the heater, which is controlled by the SLM, until the beaker arrives at a preset temperature based upon the boiling points of the analyte of interest and the solvent. After the sixty minute extraction time, the SLM lifts the thimble out of the boiling solvent for the rinse operation. After the rinsing is complete, the reflux valve closes, collecting condensing fluid in the column, reducing the volume of solvent in the beaker and concentrating the analytes in the remaining liquid. The SLM uses the video system to monitor the liquid level and, if the level drops below a specified valve, the SLM opens the reflux valve momentarily to allow some solvent back into the beaker. Once the extraction is complete and the beaker has had time to 
cool, the SLM lowers the beaker and sends a signal indicating the process is complete. The robot removes the beaker containing the extracted analytes, discards the thimble containing the original soil sample, and the process is ready to process a new soil sample.

\section{HVC SLM}

The HVC SLM automates the Kuderna-Danish evaporation process. A robot places a beaker containing the analytes dissolved in a volatile organic solvent in the input port. This beaker can be the beaker taken from the output port of the Soxhlet extractor. The beaker can be any size within a specified range. An output vial, capped with a septum, must also be provided to the output port. Once the beaker and vial are in place, no further action by the robot or analyst is needed until the process is complete. A sensor confirms the presence of the beaker and that it is an allowed size. The SLM lowers a metal tube into the beaker and siphons the solution into the condensing flask. Clean solvent is sprayed onto the sides of the beaker to rinse any residual analyte to the bottom and this rinse is also drawn into the concentration flask.

The SLM applies a user-specified partial vacuum to the concentration flask and applies heat. Heating preferentially boils off the solvent, leaving the analytes behind. Partial refluxing increases the effectiveness of separation. The vapor that passes out of the reflux column is collected in a waste-solvent container for reclamation or disposal. Solvent exchange could be effected at this point by adding a different solvent and repeating the process, but the current PCB analysis does not require a solvent exchange. Sensors determine when the solvent volume is sufficiently reduced and the concentrate is then transferred to another chamber for final volume adjustment. Rinses of the concentration flask are also transferred, assuring complete transfer of the analytes. If the volume needs to be increased, the SLM adds solvent until sensors indicate that the exact volume has been reached. If the volume needs to be reduced, the SLM evaporates additional solvent by blowing pure nitrogen against the solvent surface until the proper volume is reached. The SLM is precalibrated to deliver the final volume desired for the analysis.

In the output port, the SLM inserts a hypodermic needle through the septum on the output vial, partially evacuates the vial volume and then pumps the volume-adjusted extract through the needle into the vial. The needle is extracted and the SLM signals that the process is complete and the vial is ready for pickup. Once the vial and input beaker have been removed, the concentrator is ready for the next sample. 


\section{Column SLM}

The column SLM automates the clean-up process. The SLM is a modified version of the Gilson syringe pump and sampling injector equipment. The robot or analyst inserts the capped vial containing the sample into the SLM and provides an output vial and a fresh, prepacked clean-up column. The SLM requires no further attention from the robot or analyst until the clean-up process is complete. The clean-up SLM uses the single syringe and valve configuration of the Gilson syringe pump as a low-pressure pump for transferring liquids. The SLM uses a three-port valve to allow aspiration from the reservoir port or the needle port; dispensing occurs only through the needle port. The syringe pump is connected to a sampling injector with a Gilson serial input/output channel cable. The sampling injector is equipped with a rinsing station to clean the needle externally and internally. Since the columns are prepacked, the type of column packing is determined by which column is presented to the SLM. The PCB SAM uses prepacked Florisil columns. The SLM uses the needle to penetrate the septum sealing the input vial, and withdraws most of the sample solution. This is transferred to the clean-up column, eluted with solvent, collected and then transferred to a second vial. Since the PCBs are not retained by the Florisil, no additional solvents are added and the concentration of the analytes is not changed. If different fractions containing different classes of analytes were desired, those samples could be eluted with other solvents and the sample would have to be returned to the HVC for volume adjustment. Excess solution is discarded to a waste container. The second vial is then ready for pickup by a robot or analyst. Once the input vial, output vial, and used cartridge have been removed, the SLM signals that it is ready to receive another sample.

\section{Gas Chromatograph (GC) SLM}

Even in the manual process, once the sample is injected into the GC, nothing obvious happens until the analytes elute through the detector. Indeed, the core of a GC has no moving parts. The only operator attention required per sample is for the injection of the sample. Most current GCs are equipped with automatic injectors. In addition, almost all current GCs have some means of connecting the detector output to a dataacquisition device. Therefore, modern GCs need very little modification to become SLMs. The current GC SLM, for example, is a Varian off-the-shelf model with only software modifications required to make it a compliant SLM.

The GC SLM operation begins when a robot or analyst places a septum-capped vial in the GC autosampler tray. Upon receiving a "start" command from the TSC or the analyst, the autoinjector pierces the septum with a hypodermic needle and withdraws into a microsyringe a small amount of the sample--typically one or two microliters. The syringe moves to the injection port of the GC and injects the sample. The SLM then rinses the syringe with clean solvent. Once a robot or analyst removes the vial from the autosampler tray after the GC run is completed, the GC SLM signals that it is ready for the next sample. The detector output is transmitted to a data-acquisition device or to the DIM, described below.

The SLM GC, like most commercially available GCs, has a touch pad for user entry of parameters such as the sample-injection volume, oven temperature, changes in oven temperature during analyte separation, and temperature equilibration times after the sample run is completed. These parameters can be changed by the TSC as well as through preprogrammed methods stored within the GC. 


\section{Robot SLM}

The robot SLM transports materials needed in the chemical operations of the PCB SAM. The ORCA robot was manufactured by Hewlett Packard Corporation (in a division since sold to Sagian). Its software runs on a PC using the Windows 3.1 operating system. The robot SIMM incorporates the CAA communication package and commands can be prepared using the SLM developer's toolkit. The TSC (described below) instructs the ORCA robot SLM via its transport command to move an item from one location to another. The transport command includes parameters describing the type of item being transported, the SLM, the port, and the port index the item should be retrieved from or delivered to. The robot is able to change grippers to accommodate the different sizes of objects used in the PCB SAM.

\section{TSC}

The TSC is the software that provides the SLM coordination. From a software perspective, each analytical method is described by a "script" or list of operations that make up that method. The TSC executes these scripts by expanding them into the set of elemental operations to be performed by SLMs in the laboratory. The TSC will then select an SLM to perform each operation based on the availability of the SLM's, sample processing priorities, and any device-selection criteria specified in the script. Selection of the SLM to perform an operation is made at run time; thus, redundant SLMs may be added to the system at any time to increase sample throughput. Similarly, if one of two or more SLMs of a particular type is taken off-line or reports an error, the TSC will route samples around it. It is the responsibility of the TSC to ensure that all material transport is performed for the selected SLM and to initiate the operation of each SLM.

The TSC is able to communicate with any compliant SLM because all compliant SLMs will use a vendor-specified set of commands in a prescribed format. Common communication protocols for all commands are less challenging than might first appear because the SLMs are highly autonomous and only minimal amounts of information must be exchanged between the TSC and any SLM.

The TSC is a computer program that gives commands to other machines in a format that is transparent to an analyst. The $\mathrm{HCl}$ provides a more intuitive interface between the analyst and the TSC. The analyst understands the laboratory operation in terms of analytical methods and laboratory procedures. The $\mathrm{HCl}$ allows the analyst to use a graphical-user interface to submit samples and prescribe required laboratory procedures, plus any special sample priority, in a way that is easily understood by an analyst. Then for actual execution, the $\mathrm{HCl}$ translates these commands into instructions that are recognized by the TSC. Similarly, messages from the SLMs to the TSC, for example, ready conditions or error conditions, are passed on to the $\mathrm{HCl}$, which translates them into terms familiar to the analyst.

\section{DIM}

The DIM automates the analysis of data from the analytical instrument. It also performs quality assurance and quality-control calculations. Although the DIM is a software tool, it behaves like any other SLM, using the same communication protocols and responding to commands from the TSC. The TSC initiates the analysis of a batch of 
samples by reading the script containing the commands describing the analytical method. Information collected during sample preparation that later will be required by the DIM is written to a data base. When the analytical SLM has produced the raw data, those data are passed to the DIM to begin the data analysis.

The DIM determines the presence of analytes of interest and their concentration. The analytical method used by the DIM will depend on the analytical instrument used and the analytes of interest. Analysis of metals by atomic absorption requires simple univariant analyses, while analysis of GC chromatograms for PCBs requires complex, parallel, multivariant data analyses coupled to a fuzzy logic result fusion module.

The DIM writes the results of the analysis in any of a variety of formats. It will send the data in the format required by the system database but can also, for example, write the data in a form readable by common spreadsheet programs. Data retrieved from the database can be analyzed and examined by the operator using the DIM off-line.

\section{Developmental Test Plan}

The CAA program's developmental test objectives, for the chemical analysis of PCBs in soil and for subsequent developmental tests of other CAA modules, are as follows:

- To demonstrate that the technologies meet the chemical analysis requirements (protocols) promulgated by the U.S. EPA for the analysis of PCBs in soil using methods $3541,3620 \mathrm{~A}$, and 8082 . $^{3}$ These protocols constitute the minimum requirements for the chemical performance of the overall system.

- To verify that each of the hardware and software components and the fully integrated system have achieved the established design performance goals.

- To generate commercially useful data pertaining to the system's work flow (e.g., time expended to perform analyses, mean time between failures, and so forth) and its chemical analytical performance (ability to meet or to exceed EPA regulations). The tests should serve as a reliable predictor of areas in which the system will demonstrate increased effectiveness, reduced cost, or both.

The developmental tests are undertaken with the expectation that the system will perform according to its required capabilities. However, it is not uncommon for the tests to reveal areas in which corrections or modifications need to be made. For this reason, developmental tests are considered an early point along a testing continuum a way of establishing progress toward a stable engineering design, leading to a manufacturing program. Thus, the following should be considered additional test goals:

- To identify any technology or subsystem that is not fully meeting its stated performance objectives. Testing should identify any areas where a technology (or system) falls short of its objectives. Any deficient technology or system should not exit testing until the required capabilities can be demonstrated or other alternatives established.

- To assure that hardware and software have stabilized, so that both design risks and manufacturing and production decision uncertainties are minimized. A repeated inability during testing to meet requirements, where 
the failures are not due to inappropriate setup of the equipment or to inadequate logistical support, are an indication that a modification to design may be necessary.

The developmental test plan is organized into test clusters designed to validate both the mechanical and chemical performance of the test system. The PCB SAM developmental test plan includes the following test clusters:

- Stand-alone testing of Soxhlet SLM commands and automatic functions

- Stand-alone testing of concentrator SLM commands and automatic functions

- Stand-alone testing of column SLM commands and automatic functions

- GC detector linearity and calibration

- Stand-alone testing of GC SLM commands and automatic functions

- Chemical analytical performance in sand, loam, and clay; precision and accuracy at high- and low-concentration levels

- HCl-TSC-SLM-robot commands, functions, and communication

- DIM performance, comparison to established methods

- Work-flows, labor requirements

- Consumables, solvent waste

- Reliability

- Manuals and documentation

Predevelopmental testing of the automated PCB SAM occurred during September December 1996 at ORNL. Twenty-two environmental soil samples previously analyzed by a third-party laboratory and known to contain quantifiable amounts of PCBs were processed through the automated PCB SAM. The analytical results are discussed below. In addition, testing activity related to the developmental test plan test clusters began in April 1997. Preliminary results obtained from these tests are also discussed below. 


\section{RESULTS}

\section{Predevelopmental Testing}

During September 1996, the CAA program conducted a preliminary test of the automated PCB SAM using environmental soil samples supplied and previously analyzed by a third-party analytical laboratory. The results obtained by the third-party laboratory were generated using SW-846 methods $3540 / 3620 \mathrm{~A} / 8080$ in a manual mode of operation. A graph comparing the results obtained by the manual method versus the automated PCB SAM is shown is Figure 1.

The total PCB concentration obtained via each method of analysis is plotted against the corresponding sample identification number. The PCB concentration in these samples ranged between $0.9 \mathrm{ppm}$ and $38.7 \mathrm{ppm}$.

The graph in Figure 2 is an interactive bar chart that plots the difference in PCB concentration between the two measurement methods (Manual - CAA) versus the sample identification number.

The comparative results demonstrate good agreement between the FIGURE 1

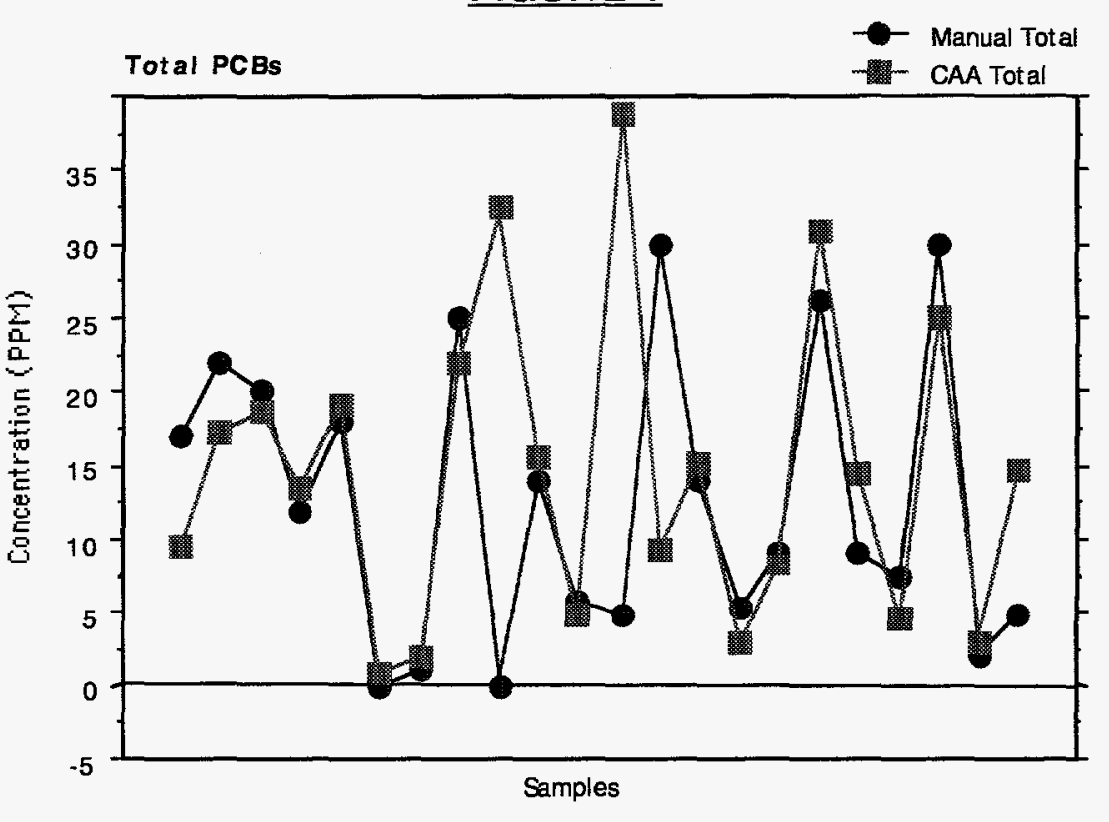

FIGURE 2 Interaction Bar Plol for Manual-CAA

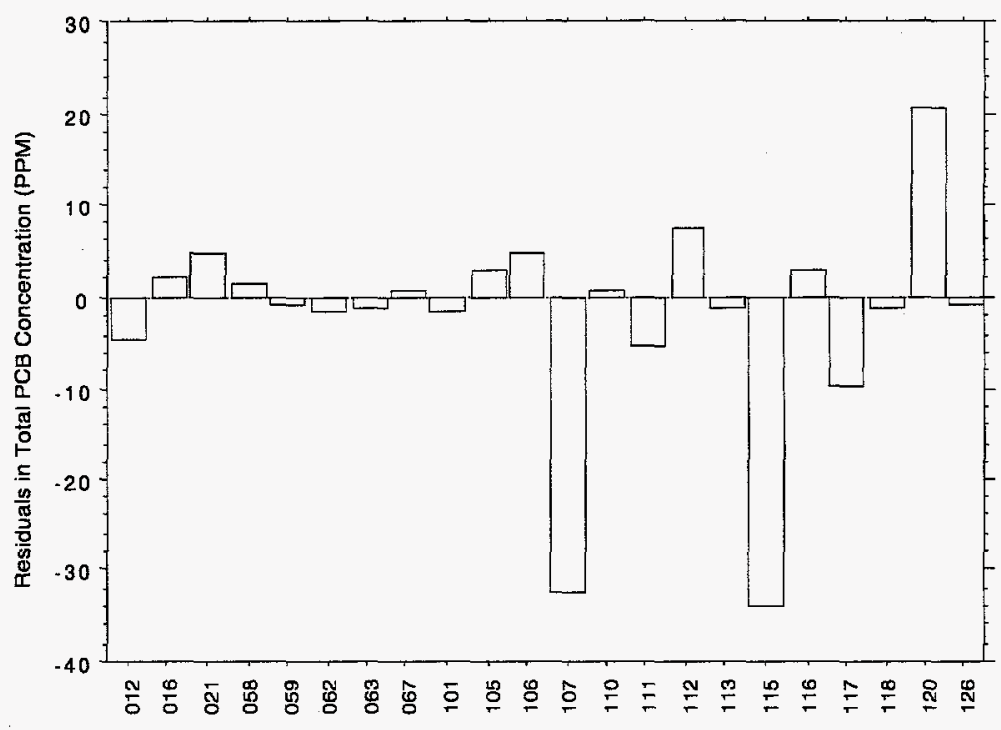

two methods of analysis. Issues such as sample homogeneity and storage history add to the uncertainty of the measurement process in both cases. 
Although the chemical performance of the PCB SAM met the expectations of the CAA program, the physical attributes of these samples brought to light some minor design deficiencies. As a result of this test, enhancements were added to the HVC to permit a two-solvent operation. This allows the user to flush and wash the system with a different, i.e. more polar, solvent between the processing of samples. This greatly eliminated cross-contamination problems encountered with samples heavily loaded with PCBs. It also increases the efficiency of removing extraneous organic materials coextracted with the PCBs from the HVC system.

A Method Detection Limit Study (MDL), as defined in Chapter One of the EPA's SW-846 methods manual, was performed using the automated PCB SAM for Aroclors 1242,1254 , and 1260. The appropriate PCB mixture was spiked at a level of 100 $\mathrm{ug} / \mathrm{kg}$ into 10 grams of sodium sulfate and processed with the PCB SAM system to a final volume of $10 \mathrm{mls}$ in hexane. In addition, a midconcentration level spike-recovery study was performed under the same conditions at a concentration level of $400 \mathrm{ug} / \mathrm{kg}$. The percent recovery, standard deviation, and experimental MDL results are listed in Table 1.

TABLE 1

MDL / MID Level Study Test Results

\begin{tabular}{|c|c|c|c|c|c|}
\hline & \multicolumn{3}{|c|}{$\overline{M D L}$ Study } & MID Level & Study \\
\hline PCB & $\%$ Recovery & Std Dev & MDL(ug/kg) & \%Recovery & Std Dev \\
\hline 1242 & 92.8 & 4.3 & 13.5 & 97.6 & 14.9 \\
\hline 1254 & 79.5 & 8.4 & 23.4 & 88.0 & 3.3 \\
\hline 1260 & 100.1 & 6.2 & 19.5 & 90.7 & 2.6 \\
\hline Mixture & & & & & \\
\hline 1254 & 93.5 & 4.4 & 13.8 & - & - \\
\hline 1260 & - & - & & 93.0 & 2.9 \\
\hline$\overline{\mathrm{T}} \overline{\mathrm{O}} \overline{\mathrm{T}} \overline{\mathrm{A}} \overline{\mathrm{L}} \overline{\mathrm{S}}$ & & & & & \\
\hline $\begin{array}{l}\text { Mean } \\
\text { Std Dev }\end{array}$ & $\begin{array}{l}91.5 \\
8.6\end{array}$ & $\begin{array}{l}5.8 \\
19\end{array}$ & - & $\begin{array}{l}92.3 \\
4.1\end{array}$ & $\begin{array}{l}5.9 \\
6.0\end{array}$ \\
\hline
\end{tabular}

\section{Developmental Testing}

As part of the precision and accuracy test cluster requirements of the developmental test plan, a series of PCB recovery studies were performed using Aroclors 1242, 1254, and 1260. In order to optimize the time and resources available for these tests, a mixture analysis procedure was statistically designed to generate the maximum amount of information in the shortest available time period (minimum number of analytical determinations needed to provide statistically relevant data). This procedure encompassed a triangle pattern where each individual PCB Aroclor is located at the intersection of each point of a triangle, with the midpoint of each line representing an equal mixture of the two Aroclors represented at each end point of that line, and the center position of the triangle representing an equal mixture of all three PCB Aroclors. This is graphically represented in Figure 3. 


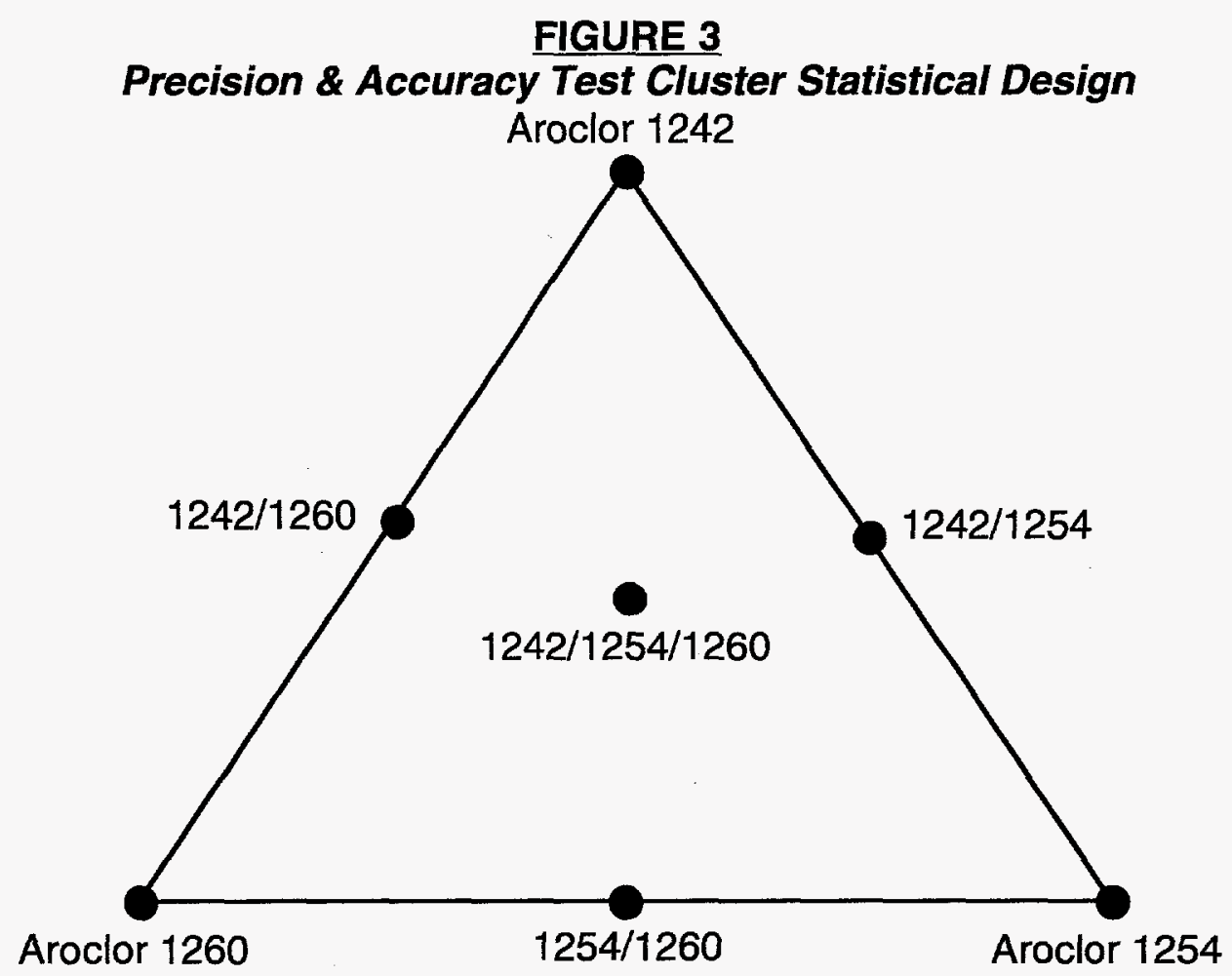

The test was performed for each matrix of interest using the same experimental conditions utilized under the predevelopmental testing MDL/MID study. Thus each test, one each for clay, loam, and sand matrices, was comprised of seven analytical determinations (three individual Aroclors, three two-Aroclor mixtures, and one threeAroclor mixture). Each matrix test was performed at two different levels of PCB concentration, $180 \mathrm{ug} / \mathrm{kg}$ and $720 \mathrm{ug} / \mathrm{kg}$, with each individual point on the triangle totaling the PCB concentration of that test. For example, for the $180 \mathrm{ug} / \mathrm{kg}$ clay matrix using a two Aroclor mixture test point, a 10-gram sample of clay was spiked at a concentration level of $90 \mathrm{ug} / \mathrm{kg}$ for each Aroclor being tested, yielding a total PCB spike-concentration level of $180 \mathrm{ug} / \mathrm{kg}$. The analytical results of these tests are listed in Table 2.

Based upon the results obtained from the precision and accuracy test cluster, an additional MDL study was performed for each matrix being tested using Aroclor 1242 at a spike concentration level of $72 \mathrm{ug} / \mathrm{kg}$. 
TABLE 2

Precision \& Accuracy Test Cluster Test Results

\begin{tabular}{|c|c|c|c|c|c|c|c|c|c|}
\hline \multirow{2}{*}{\multicolumn{2}{|c|}{$\%$ Recovery }} & \multicolumn{2}{|c|}{1242} & \multicolumn{2}{|c|}{1254} & \multicolumn{2}{|c|}{1260} & \multicolumn{2}{|c|}{ Matrix } \\
\hline & & $180 \mathrm{ppb}$ & $720 \mathrm{ppb}$ & $180 \mathrm{ppb}$ & $720 \mathrm{ppb}$ & $180 \mathrm{ppb}$ & $720 \mathrm{ppb}$ & $\frac{\text { Avg. }}{(n=24)}$ & Std Dev \\
\hline & Sand & 72.6 & 81.8 & 73.0 & 86.6 & 84.6 & 82.8 & 80.2 & 6.0 \\
\hline & Clay & 58.9 & 85.7 & 87.0 & 86.5 & 76.1 & 84.4 & 79.7 & 11.0 \\
\hline & Loam & 92.9 & 91.6 & 102.6 & 98.3 & 74.5 & 92.0 & 92.0 & 9.6 \\
\hline $\begin{array}{r}\text { Aroclor } \\
(n=12\end{array}$ & Avg: & $\overline{7} \overline{4} . \overline{8}$ & $\overline{86} \cdot \overline{3}$ & $\overline{8} \overline{7.5}$ & $\overline{90} . \overline{4}$ & $-\overline{78.4}$ & $\overline{8} \overline{6} . \overline{4}$ & 84.0 & $6.0^{---}$ \\
\hline Std & Dev: & 18.6 & 7.9 & 18.6 & 10.8 & 9.2 & 7.7 & - & - \\
\hline
\end{tabular}

This particular PCB mixture exhibited the lowest percent recovery data in the previous test. Thus, the data generated under this MDL test would signify the worst-case performance scenario for the PCB SAM system. The PCB SAM operating parameters remained the same as in the previous MDL study. The results of this test are listed in Table 3.

TABLE 3

Matrix MDL Study with Aroclor 1242

\begin{tabular}{|l|l|l|l|}
\hline Matrix $(n=8)$ & \% Recovery & Std Dev. & MDL (ug/kg) \\
\cline { 2 - 4 } $\mathrm{Na}_{2} \mathrm{SO}_{4}$ & 74.3 & 9.5 & 23.2 \\
Clay & 91.1 & 6.6 & 16.2 \\
Loam & 101.3 & 20.6 & 33.6 \\
Sand & 73.3 & 12.1 & 31.4 \\
\hline
\end{tabular}

\section{DISCUSSIONS}

The performance of the PCB SAM system has undergone rigorous chemical validation over the past 12-14 months. The predevelopmental testing of the system using PCBcontaminated soil samples demonstrated the system's ability to reproduce analytical results obtained using traditional, nonautomated manual techniques. The PCB results obtained by the PCB SAM in this study shows excellent agreement with those results obtained by the original testing laboratory, given the systemic errors typically associated with collecting and analyzing soil matrices. In addition, under both the MDL/MID study performed in the predevelopmental testing phase of this project and the MDL test performed under the development test phase using Aroclor 1242, the results demonstrate that the PCB SAM system's calculated MDLs exceed the 50-70 ug/kg method performance range listed in EPA Method 8082.

In determining how the chemical performance of the PCB SAM compares to known validated methodologies, it is helpful to compare related data collected during the chemical validation of the methools. EPA Method 8082 provides single laboratory and 
multilaboratory performance data in Tables 9 and 10 of the published method. A brief summary of these data, as percent recovery, is listed in the following tables, where Table 4 lists the summary of PCB recovery data collected in the single laboratory testing of the EPA method and Table 5 lists the summary of results from the multiple laboratory precision and accuracy study for the same EPA method.

TABLE 4

EPA Method 8082 Single Laboratory Performan
\begin{tabular}{lccc}
\multicolumn{4}{c|}{ All Levels } \\
\cline { 2 - 4 } & $\underline{n}$ & Mean & Std Dev \\
Lab1 & 12 & 84.4 & 26 \\
Lab2 & 24 & 67 & 13.3 \\
Lab3 & 12 & 66 & 9.1 \\
Lab4 & 24 & 110.5 & 28.5 \\
Lab5 & 12 & 83.5 & 10.3 \\
Lab6 & 12 & 125.4 & 18.4 \\
Lab7 & 12 & 99.9 & 19 \\
Lab8 & 12 & -12 & 29.1 \\
\hline All Labs & 120 & 87.6 & 29.7 \\
\hline
\end{tabular}

TABLE 5

EPA Method 8082 Multiple Laboratory Performance Data ${ }^{5}$

\begin{tabular}{|rccccccc|}
\hline Aroclor & \multicolumn{4}{c}{1254} & & \multicolumn{3}{c|}{1260} \\
\cline { 2 - 4 } \cline { 6 - 8 } PPM & 5 & 50 & 500 & & 5 & 50 & 500 \\
All Labs & & & & & & & \\
$\mathrm{n}$ & 20 & 30 & 9 & & 21 & 31 & 9 \\
Mean & 98.8 & 92.5 & 71.3 & & 95.5 & 78.6 & 75.3 \\
Std Dev & 28.7 & 42.9 & 14.1 & & 25.3 & 18 & 9.5 \\
\hline
\end{tabular}

If one plots the percent recovery of $\mathrm{PCB}$ against the standard deviation for that measurement for all the collected data, one can make a visual comparison of the performance of the PCB SAM relative to EPA Method 8082. Such a plot is shown in Figure 4.

These data indicate excellent chemical performance comparability between the $\mathrm{PCB}$ SAM and EPA Method 8082 test data. In fact, in most cases the standard deviations resulting from the PCB SAM measurements are more tightly clustered and less than those from the data set manually generated by EPA Method 8082 . 
FIGURE 4

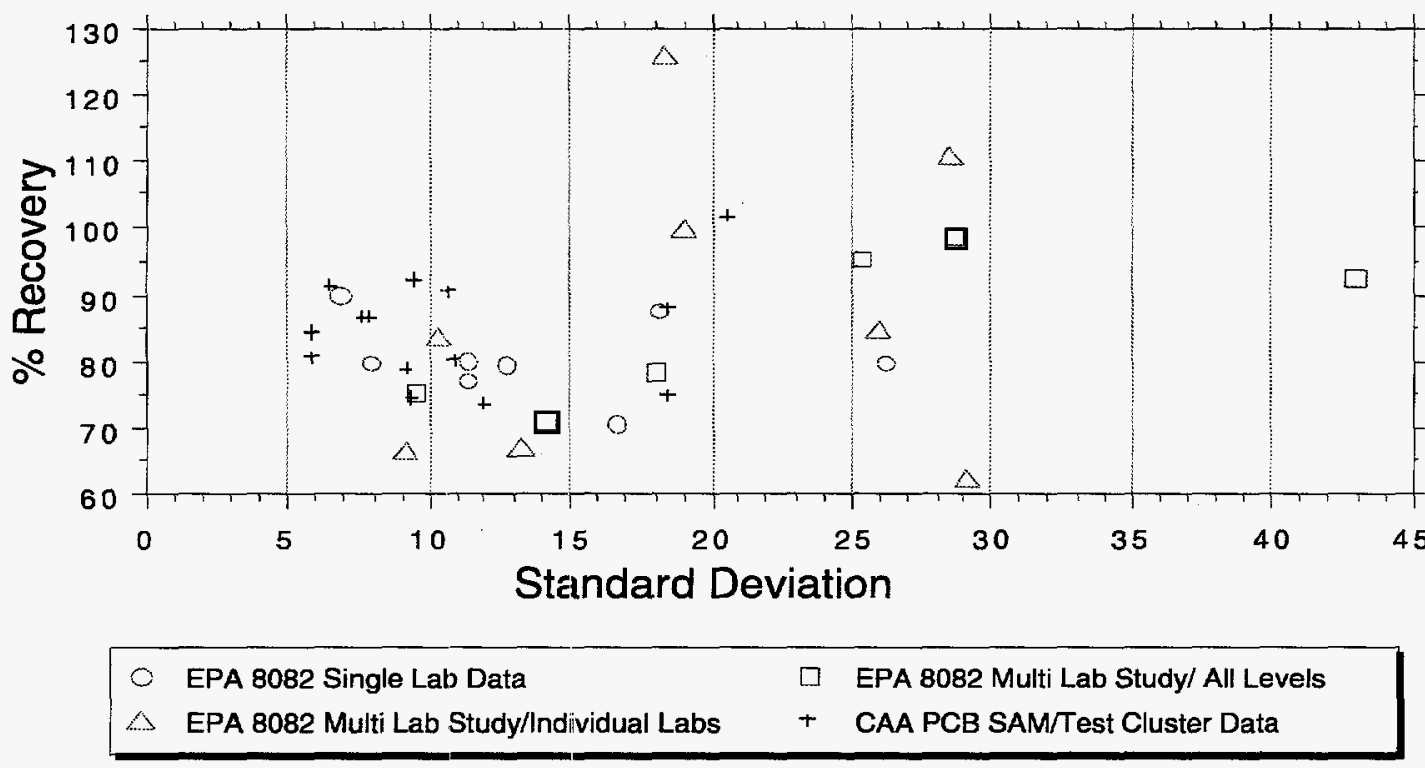

The data collected to date on the chemical performance of the PCB SAM system clearly indicates the CAA program's success in meeting several of its goals to

- reduce variations in quality among laboratories, and

- increase precision and accuracy of results.

Further developmental testing of the PCB SAM system will continue over the next three to six months in an effort to collect system performance data such as work flows, labor requirements, consumable usage, and mechanical performance data. The PCB SAM will be used during the characterization phase of a DOE environmental restoration project at ORNL. Approximately 260 surface soil samples will be collected and analyzed for PCBs with the PCB SAM. This project will form the actual basis from which the remaining developmertal test plan performance data will be collected. 


\section{ACKNOWLEDGMENTS}

The authors would like to acknowledge the help given by the following individuals:

Oak Ridge National Laboratory

James Younkin

David Thompson

Idaho National Engineering and Environmental Laboratory

Michael Clark

Terry Turner

Robert Kinoshita

Sandia National Laboratory

Jill Rivera

University of Tennessee

Martin Hunt

Institute for Defense Analyses

Rebecca Rubin

Ivan Oelrich

\section{REFERENCES}

${ }^{1}$ R. Hollen, "Automating the Analytical Laboratory via the Chemical Analysis Automation (CAA)

Program," ISLAR '97, Boston, MA, October 1997.

${ }^{2}$ Huber, Ludwig, "Validation of Computerized Analytical Systems," pp. 64-67.

3 U.S. Environmental Protection Agency, "Test Methods for Evaluating Solid Waste

Physical/Chemical Methods (SW-846)," Third Edition, Update III.

${ }^{4}$ Stewart, J.H., Bayne, C.K., Holmes, R.L., Rogers, W.F., and Maskarinec, M.P., "Evaluation of a Rapid Quantitative Organic Extraction System for Determining the Concentration of PCB in Soils," Proceedings of the U.S. EPA Symposium on Waste Testing and Quality Assurance, Oak Ridge National Laboratory, Oak Ridge, TN, 37831, July 11-15, 1988.

${ }^{5}$ Stewart, J., "EPA Verification Experiment for Validation of the SOXTECTM PCB Extraction

Procedure," Oak Ridge National Laboratory, Oak Ridge, TN, 37831-6138, October 1988. 